\title{
INTERFACE CRACKS IN ANISOTROPIC DISSIMILAR MATERIALS: GENERAL CASE
}

BY

\section{NI AND S. NEMAT-NASSER}

Center of Excellence for Advanced Materials, Department of Applied Mechanics and Engineering Sciences, University of California, San Diego, La Jolla, California

\begin{abstract}
An interface crack in anisotropic dissimilar materials is considered for the general case where all three fracture modes may be coupled. Analytic solutions are obtained for two commonly used models of interface cracks: (1) the fully open crack; and (2) the Comninou model where, even under farfield tension normal to the crack face, the existence of very small contact zones near the crack tips is permitted. Conditions under which nonoscillatory singular solutions may exist, are discussed.
\end{abstract}

1. Introduction. In a recent paper, $\mathrm{Ni}$ and Nemat-Nasser [4] presented analytic solutions for interface cracks in anisotropic half-spaces consisting of dissimilar materials, assuming a special arrangement of the anisotropy axes of the two materials, so that only Modes I and II are activated under in-plane farfield tractions: a plane problem with zero antiplane displacement component. Two models were considered: one with a fully-open crack, the other allowing contact zones near the crack tips, i.e., the Comninou model (Comninou [1]). Relations between the solutions were discussed, and the results were compared with those for the isotropic case (Gautesen and Dundurs [2,3]), as well as the anisotropic case (Qu and Bassani [5]).

In the present paper we consider the general case of two anisotropic half-spaces joining on the plane $x_{2}=0$, and containing an interface crack on the $\left(x_{1}, x_{3}\right)$ plane, with crack edges being parallel to the $x_{3}$-axis. No special arrangement of the anisotropy axes is assumed. Thus, all three fracture modes are present and coupled. It is shown that the basic singular integral equations governing the general solution involve a total of nine dimensionless parameters, denoted by $\alpha_{1}$ to $\alpha_{9}$. The nature of the singularity at the crack tips is discussed in relation to these parameters, and conditions under which nonoscillatory singular solutions can exist are examined.

Again, two commonly used models of interface cracks are considered: a fully-open crack model and the Comninou model, where the existence of contact zones near the crack tips is permitted. For the fully-open crack model, the complete analytic solution for this general case is obtained. In general, oscillatory square-root singularities are involved in the solutions at the crack tips. A necessary and sufficient condition under

Received October 24, 1990.

The first author is on leave from Academia Sinica, Beijing, China.

(C)1992 Brown University 
which nonoscillatory solutions exist for any combination of the farfield tractions is that three of the nine parameters, i.e., $\alpha_{4}, \alpha_{6}, \alpha_{8}$, are zero, which is equivalent to the vanishing of the antisymmetric matrix $\mathbf{W}$ (Ting [7], and Qu and Bassani [5]). Its physical meaning is also discussed. For the Comninou model, it is interesting to note that, unlike the case discussed in our previous paper [4], for the general case the oscillatory singularities at the crack tips cannot be removed by the introduction of contact zones without additional special assumptions. The necessary and sufficient condition under which nonoscillatory singularities exist for any combination of the farfield tractions is that the dimensionless parameter $\alpha_{6}$ be zero. The closed-form solution for this nonoscillatory generalized Comninou model is given.

2. Formulation. The formulation of the basic problem follows Willis [10], and $\mathrm{Ni}$ and Nemat-Nasser [4]. A composite of two anisotropic elastic half-spaces joining along the $x_{2}=0$ plane is considered. The elasticity tensors are $c_{i j k l}^{+}$and $c_{i j k l}^{-}$, for $x_{2}>0$ and $x_{2}<0$, respectively. All field variables are functions of $x_{1}$ and $x_{2}$ only, with the $x_{1}$-axis on the interface. Equilibrium requires

$$
\sigma_{j \beta, \beta}=c_{j \beta k \alpha} u_{k, \alpha \beta}=0 \quad x_{2} \neq 0
$$

with $j, k=1,2,3$, and $\alpha, \beta=1,2$; a comma followed by an index denotes partial differentiation with respect to the corresponding coordinate variable; and repeated indices are summed. Here, $\mathbf{u}$ and $\sigma$ denote the displacement and stress fields, respectively. The boundary conditions are

$$
\sigma_{j 2}=c_{j 2 k \beta}^{+} u_{k, \beta}\left(x_{1}, 0^{+}\right)=c_{j 2 k \beta}^{-} u_{k, \beta}\left(x_{1}, 0^{-}\right)=t_{j}\left(x_{1}\right) \text {, }
$$

with the crack opening given by

$$
\mathbf{u}\left(x_{1}, 0^{+}\right)-\mathbf{u}\left(x_{1}, 0^{-}\right) \equiv \mathbf{b}\left(x_{1}\right)=-\int_{-\infty}^{x_{1}} \mathbf{B}(\xi) d \xi,
$$

where $\mathbf{B}\left(x_{1}\right)$ is the dislocation density vector. In addition, we require that

$$
\mathbf{u}\left(x_{1}, x_{2}\right) \rightarrow 0 \text { as }\left|x_{2}\right| \rightarrow \infty \text {. }
$$

From [4], the displacement and stress fields for the above-stated problem are given by

$$
\begin{aligned}
\mathbf{u}\left(x_{1}, x_{2}\right) & =\frac{\operatorname{sgn}\left(x_{2}\right)}{\pi} \Gamma\left\{\operatorname{Im}\left[\mathbf{M}_{0} \mathbf{L}^{-1}\right]_{ \pm} *\left[\int_{-\infty}^{x_{1}} \mathbf{t}(\xi) d \xi-\int_{x_{1}}^{\infty} \mathbf{t}(\xi) d \xi\right]\right\}, \\
\boldsymbol{\tau}_{1}\left(x_{1}, x_{2}\right) & =\frac{\operatorname{sgn}\left(x_{2}\right)}{\pi}(\mathbf{I}-\boldsymbol{\Gamma})\left\{\operatorname{Im}\left[\mathbf{N} \mathbf{M}_{0} \mathbf{L}^{-1}\right]_{ \pm} * \mathbf{t}\left(x_{1}\right)\right\}, \\
\boldsymbol{\tau}_{2}\left(x_{1}, x_{2}\right) & =-\frac{\operatorname{sgn}\left(x_{2}\right)}{\pi}(\mathbf{I}-\boldsymbol{\Gamma})\left\{\operatorname{Im}\left[\mathbf{M}_{0} \mathbf{L}^{-1}\right]_{ \pm} * \mathbf{t}\left(x_{1}\right)\right\} \\
\boldsymbol{\tau}_{3}\left(x_{1}, x_{2}\right) & =-\frac{\operatorname{sgn}\left(x_{2}\right)}{\pi} \operatorname{Im}\left[(\mathbf{U} \boldsymbol{\Gamma}+\mathbf{V} \boldsymbol{\Gamma} \mathbf{N}) \mathbf{M}_{0} \mathbf{L}^{-1}\right]_{ \pm} * \mathbf{t}\left(x_{1}\right),
\end{aligned}
$$

where $*$ denotes the convolution integral. The quantities in these equations are defined as follows: $\boldsymbol{\tau}_{i}$ with components $\left(\tau_{i}\right)_{j}, i, j=1,2,3$, defines the stress field, and $\mathbf{N}$ is a real 6 by 6 matrix given by

$$
\mathbf{N}=-\left[\begin{array}{cc}
\mathbf{T}^{-1} \mathbf{R}^{\mathrm{T}} & \mathbf{T}^{-1} \\
\mathbf{R} \mathbf{T}^{-1} \mathbf{R}^{\mathrm{T}}-\mathbf{Q} & \mathbf{R} \mathbf{T}^{-1}
\end{array}\right]
$$


where

$$
\mathbf{Q}=\left[c_{j 1 k 1}\right], \quad \mathbf{R}=\left[c_{j 1 k 2}\right], \quad \mathbf{T}=\left[c_{j 2 k 2}\right] ;
$$

the eigenvalues of the real matrix $\mathbf{N}$ are all complex. We denote the first three eigenvalues with positive imaginary parts by $p_{a}(a=1,2,3)$, and the corresponding eigenvectors by

$$
\left[\begin{array}{l}
\mathbf{a}_{b} \\
\mathbf{l}_{b}
\end{array}\right], \quad b=1,2,3,
$$

where $\mathbf{a}_{b}, \mathbf{l}_{b}$ are three-dimensional vectors. The matrices $\mathbf{U}$ and $\mathbf{V}$ are defined by

$$
\mathbf{U}=\left[c_{i 3 k 1}\right], \quad \mathbf{V}=\left[c_{i 3 k 2}\right],
$$

and $\boldsymbol{\Gamma}$ is a projection operator, which selects the first three components of its argument. The 6 by 3 matrix $M_{0}$ is defined by

$$
\mathbf{M}_{0}=\sum_{a=1}^{3}\left[\frac{\mathbf{I}}{x_{1}+p_{a} x_{2}}-\frac{x_{2}\left(\mathbf{N}-p_{a} \mathbf{I}\right)}{\left(x_{1}+p_{a} x_{2}\right)^{2}}+\frac{x_{2}^{2}\left(\mathbf{N}-p_{a} \mathbf{I}\right)^{2}}{\left(x_{1}+p_{a} x_{2}\right)^{3}}\right]\left[\begin{array}{l}
\mathbf{A}_{a} \\
\mathbf{L}_{a}
\end{array}\right]
$$

Finally, the quantities $\mathbf{A}_{b}, \mathbf{L}_{b}$, and $\mathbf{L}$ in (2.3) and (2.4e) are defined by

$$
\begin{aligned}
\mathbf{A}_{b} & =\left[\mathbf{a}_{1} \delta_{1 b}, \mathbf{a}_{2} \delta_{2 b}, \mathbf{a}_{3} \delta_{3 b}\right], \\
\mathbf{L}_{b} & =\left[\mathbf{I}_{1} \delta_{1 b}, \mathbf{I}_{2} \delta_{2 b}, \mathbf{I}_{3} \delta_{3 b}\right], \\
\mathbf{L} & =\left[\mathbf{I}_{1}, \mathbf{I}_{2}, \mathbf{I}_{3}\right] .
\end{aligned}
$$

The displacement and stress fields may also be expressed in terms of the dislocation density vector $\mathbf{B}\left(x_{1}\right)$ by

$$
\begin{aligned}
\mathbf{u}\left(x_{1}, x_{2}\right) & =\frac{1}{\pi} \boldsymbol{\Gamma}\left[\operatorname{Re}\left(\mathbf{M}_{0} \mathbf{L}^{-1} \boldsymbol{\Lambda}\right)_{ \pm} * \int_{-\infty}^{x_{1}} \mathbf{B}(\xi) d \xi\right], \\
\boldsymbol{\tau}_{1}\left(x_{1}, x_{2}\right) & =-\frac{1}{\pi}(\mathbf{I}-\boldsymbol{\Gamma})\left[\operatorname{Re}\left(\mathbf{N M}_{0} \mathbf{L}^{-1} \boldsymbol{\Lambda}\right)_{ \pm} * \mathbf{B}\left(x_{1}\right)\right], \\
\boldsymbol{\tau}_{2}\left(x_{1}, x_{2}\right) & =\frac{1}{\pi}(\mathbf{I}-\boldsymbol{\Gamma})\left[\operatorname{Re}\left(\mathbf{M}_{0} \mathbf{L}^{-1} \boldsymbol{\Lambda}\right)_{ \pm} * \mathbf{B}\left(x_{1}\right)\right], \\
\boldsymbol{\tau}_{3}\left(x_{1}, x_{2}\right) & =-\frac{1}{\pi} \operatorname{Re}\left[(\mathbf{U} \boldsymbol{\Gamma}+\mathbf{V} \boldsymbol{\Gamma} \mathbf{N}) \mathbf{M}_{0} \mathbf{L}^{-1} \boldsymbol{\Lambda}\right]_{ \pm} * \mathbf{B}\left(x_{1}\right),
\end{aligned}
$$

where the positive-definite matrix $\Lambda$ is defined by

$$
\boldsymbol{\Lambda}=-i\left[\overline{\mathbf{A}}_{+} \overline{\mathbf{L}}_{+}^{-1}-\mathbf{A}_{-} \mathbf{L}_{-}^{-1}\right]^{-1} \text {, }
$$

with

$$
\mathbf{A}=\left[\mathbf{a}_{1}, \mathbf{a}_{2}, \mathbf{a}_{3}\right]
$$

and $\boldsymbol{\Lambda}_{-}=\boldsymbol{\Lambda}, \boldsymbol{\Lambda}_{+}=\overline{\boldsymbol{\Lambda}}$.

The relation between the dislocation density $\mathbf{B}\left(x_{1}\right)$ and the interface tractions $\mathbf{t}\left(x_{1}\right)$ is (Willis [10])

$$
\mathbf{t}\left(x_{1}\right)=\frac{1}{\pi} \operatorname{Re}\left[\frac{\Lambda}{x_{1}-0 i}\right] * \mathbf{B}\left(x_{1}\right) .
$$

This relation is basic to our discussion. We combine (2.7) with proper boundary conditions over the crack surfaces, to obtain the dislocation density $\mathbf{B}\left(x_{1}\right)$, from which the displacement and stress fields are obtained. 
3. Interface crack models. In [4] special cases are considered where the in-plane and anti-plane deformations are decoupled. In the present work, we consider the general case where all three fracture modes are coupled. The dislocation density $\mathbf{B}\left(x_{1}\right)=\left(B_{1}\left(x_{1}\right), B_{2}\left(x_{1}\right), B_{3}\left(x_{1}\right)\right)^{\mathrm{T}}$ then has three nontrivial components.

Here, again, we examine two interface crack models: (i) the fully-open model and (ii) the Comninou model.

3.1. The fully-open crack model. Let an interface crack on the $\left(x_{1}, x_{3}\right)$-plane extend from $x_{1}=-a$ to $x_{1}=b$, with crack edges being parallel to the $x_{3}$-axis. Normal tractions $T\left(x_{1}\right)$, in-plane shear tractions $S\left(x_{1}\right)$, and anti-plane shear tractions $J\left(x_{1}\right)$ are applied far from the interface crack in the $x_{2^{-}}, x_{1^{-}}$, and $x_{3}$-direction, respectively.

Since all field variables are functions of $x_{1}$ and $x_{2}$ only, in the sequel we consider the $\left(x_{1}, x_{2}\right)$-plane at $x_{2}=0$. When the crack is fully open, the total tractions on the crack surface $[-a, b]$ are zero. From these boundary conditions and relations (2.7), it follows that

$$
\frac{1}{\pi} \operatorname{Re}\left[\frac{\boldsymbol{\Lambda}}{x_{1}-0 i}\right] * \mathbf{B}\left(x_{1}\right)+\boldsymbol{\tau}\left(x_{1}\right)=0, \quad \text { for }-a<x_{1}<b,
$$

where $\boldsymbol{\tau}\left(x_{1}\right)=\left(S\left(x_{1}\right), T\left(x_{1}\right), J\left(x_{1}\right)\right)^{\mathrm{T}}$.

The remaining boundary conditions follow from the fact that outside the crack zone, the gap, the tangential shift along the $x_{1}$-axis, and the anti-plane shift along the $x_{3}$-axis are all zero. We therefore have

$$
\int_{-a}^{b} \mathbf{B}(\xi) d \xi=0, \quad \text { and } \mathbf{B}\left(x_{1}\right)=0 \text { for } x_{1}<-a, x_{1}>b .
$$

Employing a method similar to that used in [4], subject to conditions $(3.2 \mathrm{a}, \mathrm{b})$, the system of singular integral equations (3.1) is solved analytically for the three components of the dislocation density vector $\mathbf{B}\left(x_{1}\right)$, as discussed in Sec. 4 .

For the fully-open crack model, the solutions for $\mathbf{B}\left(x_{1}\right)$ and $\mathbf{t}\left(x_{1}\right)$ are, in general, oscillatorily singular, as $x_{1} \rightarrow-a$ or $b$.

3.2. The Comninou model. Let the interface crack on the $x_{1}$-axis extend from $-L$ to $L(L>0)$, and uniform tractions $\tau=(S, T, J)$ be applied far from the interface crack. Assume that the crack is open and traction-free over the interval $(-a, b)$. Over the contact zones $(-L,-a)$ and $(b, L)$, the crack is closed. Let the contact be frictionless. Then, the tangential tractions in the $x_{1}$ - and $x_{3}$-directions vanish over the interval $(-L, L)$. Therefore, we have the following equation for $\mathbf{B}\left(x_{1}\right)$ :

$$
\left[\begin{array}{ccc}
1 & 0 & 0 \\
0 & H\left[\left(x_{1}+a\right)\left(b-x_{1}\right)\right] & 0 \\
0 & 0 & 1
\end{array}\right]\left\{\frac{1}{\pi} \operatorname{Re}\left[\frac{\boldsymbol{\Lambda}}{x_{1}-0 i}\right] * \mathbf{B}\left(x_{1}\right)+\boldsymbol{\tau}\right\}=0
$$

for $\left|x_{1}\right|<L$, where

$$
\begin{aligned}
& B_{1}\left(x_{1}\right)=B_{3}\left(x_{1}\right)=0, \quad \text { for }\left|x_{1}\right|>L, \\
& B_{2}\left(x_{1}\right)=0, \quad \text { for } x_{1}<-a \text { or } x_{1}>b, \\
& \int_{-L}^{L} B_{1}(\xi) d \xi=\int_{-L}^{L} B_{3}(\xi) d \xi=0, \quad \int_{-a}^{b} B_{2}(\xi) d \xi=0 .
\end{aligned}
$$


4. Complete solution for the fully-open crack model. We now solve the system of singular integral equations (3.1), under conditions (3.2a,b). Set

$$
\mathbf{\Lambda}=\left[\alpha_{i j}\right], \quad i, j=1,2,3,
$$

and, since $\boldsymbol{\Lambda}$ is positive-definite, introduce the additional simplifying notation:

$$
\begin{aligned}
& \alpha_{1}=\alpha_{11}>0, \quad \alpha_{2}=\alpha_{22}>0, \quad \alpha_{9}=\alpha_{33}>0, \\
& \alpha_{12}=\bar{\alpha}_{21}=\alpha_{3}+i \alpha_{4}, \quad \alpha_{13}=\bar{\alpha}_{31}=\alpha_{5}+i \alpha_{6}, \quad \alpha_{23}=\bar{\alpha}_{32}=\alpha_{7}+i \alpha_{8} .
\end{aligned}
$$

Then, setting

$$
\boldsymbol{\Lambda}_{1}=\operatorname{Re} \boldsymbol{\Lambda}=\left[\begin{array}{lll}
\alpha_{1} & \alpha_{3} & \alpha_{5} \\
\alpha_{3} & \alpha_{2} & \alpha_{7} \\
\alpha_{5} & \alpha_{7} & \alpha_{9}
\end{array}\right], \quad \boldsymbol{\Lambda}_{2}=-\operatorname{Im} \boldsymbol{\Lambda}=\left[\begin{array}{ccc}
0 & -\alpha_{4} & -\alpha_{6} \\
\alpha_{4} & 0 & -\alpha_{8} \\
\alpha_{6} & \alpha_{8} & 0
\end{array}\right],
$$

we have

$$
\Lambda=\Lambda_{1}-i \Lambda_{2}
$$

where $\Lambda_{1} \pm i \Lambda_{2}$ and $\Lambda_{1}$ are positive-definite matrices. $\boldsymbol{\Lambda}$ can also be expressed as

$$
\mathbf{\Lambda}=(\mathbf{D}+i \mathbf{W})^{-1}
$$

where $\mathbf{D}$ and $\mathbf{W}$ are real matrices introduced by Ting [7].

Using (3.2a,b) and (4.3), we write Eq. (3.1) as

$$
\frac{1}{\pi} \Lambda_{1} \int_{-a}^{b} \frac{\mathbf{B}(\xi)}{x_{1}-\xi} d \xi+\Lambda_{2} \mathbf{B}\left(x_{1}\right)+\tau\left(x_{1}\right)=0 .
$$

Since $\Lambda_{1}$ is positive-definite, we can apply $-i \Lambda_{1}^{-1}$ to both sides of (4.4) to obtain

$$
\frac{1}{i \pi} \int_{-a}^{b} \frac{\mathbf{B}(\xi)}{x_{1}-\xi} d \xi+\left(-i \mathbf{\Lambda}_{1}^{-1} \boldsymbol{\Lambda}_{2}\right) \mathbf{B}\left(x_{1}\right)-i \Lambda_{1}^{-1} \boldsymbol{\tau}\left(x_{1}\right)=0
$$

for $-a<x_{1}<b$.

One way to solve (4.5) is to diagonalize the coefficient matrix $-i \Lambda_{1}^{-1} \Lambda_{2}$ and decouple the system of integral equations to three independent integral equations.

Since $\Lambda_{1}$ is real and symmetric, and $\Lambda_{2}$ is real and anti-symmetric, the characteristic polynomial of $-i \Lambda_{1}^{-1} \Lambda_{2}$ can be written as

$$
\begin{aligned}
\left|-i \mathbf{\Lambda}_{1}^{-1} \boldsymbol{\Lambda}_{2}-\mu \mathbf{I}\right| & =-\left|\mathbf{\Lambda}_{1}^{-1}\right|\left|\mu \boldsymbol{\Lambda}_{1}+i \boldsymbol{\Lambda}_{2}\right| \\
& =-\left|\mathbf{\Lambda}_{1}^{-1}\right|\left[\mu^{3}\left|\boldsymbol{\Lambda}_{1}\right|+\mu\left(|\mathbf{\Lambda}|-\left|\boldsymbol{\Lambda}_{1}\right|\right)\right] .
\end{aligned}
$$

Therefore, the three eigenvalues of $-i \Lambda_{1}^{-1} \Lambda_{2}$ are $\mu_{1}=\lambda, \mu_{2}=-\lambda$, and $\mu_{3}=0$, with

$$
\lambda=\left(1-|\boldsymbol{\Lambda}| /\left|\boldsymbol{\Lambda}_{1}\right|\right)^{1 / 2} \text {. }
$$

It can be shown that all the eigenvalues of $-i \Lambda_{1}^{-1} \Lambda_{2}$ are greater than -1 . Therefore, $\lambda$ is real and $\left|\boldsymbol{\Lambda}_{1}\right|>|\boldsymbol{\Lambda}|$. Since $\Lambda_{1}$ is real and positive-definite, it follows that $\left|\boldsymbol{\Lambda}_{1}\right|=|\boldsymbol{\Lambda}|$ if and only if $\boldsymbol{\Lambda}_{2}=0$. In other words, $\mu_{1}=\mu_{2}=\mu_{3}=0$ if and only if $\left|\boldsymbol{\Lambda}_{2}\right|=0$, i.e., when $\alpha_{4}=\alpha_{6}=\alpha_{8}=0$. As is seen later, the vanishing of these three 
dimensionless parameters guarantees the existence of a nonoscillatory square-root singular stress field at the crack tip for the fully-open model.

Before solving Eq. (4.5), we shall briefly discuss the relations between matrices $\boldsymbol{\Lambda}_{1}, \boldsymbol{\Lambda}_{2}$ and $\mathbf{W}, \mathbf{D}$. Because $\lambda<1$, the inverse $\left(\mathbf{I} \pm i \boldsymbol{\Lambda}_{1}^{-1} \boldsymbol{\Lambda}_{2}\right)^{-1}$ exists. Then from $(4.3 \mathrm{~d})$ and

$$
\left(\mathbf{I}+i \boldsymbol{\Lambda}_{1}^{-1} \boldsymbol{\Lambda}_{2}\right)\left(\mathbf{I}-i \boldsymbol{\Lambda}_{1}^{-1} \boldsymbol{\Lambda}_{2}\right)=\mathbf{I}+\left(\boldsymbol{\Lambda}_{1}^{-1} \boldsymbol{\Lambda}_{2}\right)^{2},
$$

it follows that

$$
\begin{aligned}
\mathbf{D}+i \mathbf{W} & =\left(\boldsymbol{\Lambda}_{1}-i \boldsymbol{\Lambda}_{2}\right)^{-1}=\left(\mathbf{I}-i \boldsymbol{\Lambda}_{1}^{-1} \boldsymbol{\Lambda}_{2}\right)^{-1} \boldsymbol{\Lambda}_{1}^{-1} \\
& =\left(\mathbf{I}+i \boldsymbol{\Lambda}_{1}^{-1} \boldsymbol{\Lambda}_{2}\right)\left[\mathbf{I}+\left(\boldsymbol{\Lambda}_{1}^{-1} \boldsymbol{\Lambda}_{2}\right)^{2}\right]^{-1} \boldsymbol{\Lambda}_{1}^{-1}
\end{aligned}
$$

Therefore,

$$
\mathbf{D}=\left[\mathbf{I}+\left(\boldsymbol{\Lambda}_{1}^{-1} \boldsymbol{\Lambda}_{2}\right)^{2}\right]^{-1} \boldsymbol{\Lambda}_{1}^{-1}, \quad \mathbf{W}=\boldsymbol{\Lambda}_{1}^{-1} \boldsymbol{\Lambda}_{2}\left[\mathbf{I}+\left(\boldsymbol{\Lambda}_{1}^{-1} \boldsymbol{\Lambda}_{2}\right)^{2}\right]^{-1} \boldsymbol{\Lambda}_{1}^{-1},
$$

and

$$
\boldsymbol{\Lambda}_{1}^{-1} \boldsymbol{\Lambda}_{2}=\mathbf{W D}^{-1}, \quad \boldsymbol{\Lambda}_{1}=\left(\mathbf{D}+\mathbf{W D}^{-1} \mathbf{W}\right)^{-1} ;
$$

see also $\mathrm{Wu}$ [11]. Equation (4.5) may also be expressed as

$$
\frac{1}{i \pi} \int_{-a}^{b} \frac{\mathbf{B}(\xi)}{x_{1}-\xi} d \xi+\left(-i \mathbf{W} \mathbf{D}^{-1}\right) \mathbf{B}\left(x_{1}\right)-i\left(\mathbf{D}+\mathbf{W D}^{-1} \mathbf{W}\right) \boldsymbol{\tau}\left(x_{1}\right)=0
$$

for $-a<x_{1}<b$. Furthermore, $\lambda$, as defined in (4.6b), coincides with

$$
\beta=\left[-\frac{1}{2} \operatorname{tr}\left(\mathbf{W D}^{-1}\right)^{2}\right]^{1 / 2},
$$

which is given by Ting [7]. We can express $\lambda$ as

$$
\lambda=\left[\frac{\left|\alpha_{4} W_{12}+\alpha_{6} W_{13}+\alpha_{8} W_{23}\right|}{\left|\alpha_{1} D_{11}+\alpha_{3} D_{12}+\alpha_{5} D_{13}-\alpha_{8} W_{23}\right|}\right]^{1 / 2} .
$$

For monoclinic materials,

$$
\lambda=\sqrt{\frac{\left|\alpha_{4} W_{12}\right|}{\left|\alpha_{1} D_{11}+\alpha_{3} D_{12}\right|}}=\alpha_{4} / \sqrt{\alpha_{1} \alpha_{2}-\alpha_{3}^{2}} .
$$

Now, unless $\boldsymbol{\Lambda}_{2}=0$, there are three distinct eigenvalues for $-i \boldsymbol{\Lambda}_{1}^{-1} \boldsymbol{\Lambda}_{2}$. Therefore, $-i \Lambda_{1}^{-1} \Lambda_{2}$ can be diagonalized by a nonsingular matrix

$$
\mathbf{E}=\left[\mathbf{v}_{1}, \mathbf{v}_{2}, \mathbf{v}_{3}\right],
$$

where $\mathbf{v}_{j}$ are the eigenvectors of $-i \Lambda_{1}^{-1} \Lambda_{2}$ corresponding to the eigenvalues $\lambda_{j} \quad(j=$ $1,2,3)$, such that

$$
\mathbf{E}^{-1}\left(-i \boldsymbol{\Lambda}_{1}^{-1} \boldsymbol{\Lambda}_{2}\right) \mathbf{E}=\left[\begin{array}{ccc}
\lambda & 0 & 0 \\
0 & -\lambda & 0 \\
0 & 0 & 0
\end{array}\right]
$$


where $\lambda=\lambda_{1}=-\lambda_{2}$. The vectors $\mathbf{v}_{j}, j=1,2,3$, in $\mathbf{E}$ are given by

$$
\begin{aligned}
& \mathbf{v}_{1}=\left[\begin{array}{c}
\lambda^{2}\left(\alpha_{5} \alpha_{7}-\alpha_{3} \alpha_{9}\right)-i \lambda\left(\alpha_{6} \alpha_{7}-\alpha_{5} \alpha_{8}-\alpha_{4} \alpha_{9}\right)+\alpha_{6} \alpha_{8} \\
\lambda^{2}\left(\alpha_{1} \alpha_{9}-\alpha_{5}^{2}\right)-\alpha_{6}^{2} \\
\lambda^{2}\left(\alpha_{3} \alpha_{5}-\alpha_{1} \alpha_{7}\right)+i \lambda\left(\alpha_{3} \alpha_{6}-\alpha_{4} \alpha_{5}-\alpha_{1} \alpha_{8}\right)+\alpha_{4} \alpha_{6}
\end{array}\right] \\
&= \lambda|\boldsymbol{\Lambda}|\left[\begin{array}{c}
\lambda D_{12}-i W_{12} \\
\lambda D_{22} \\
\lambda D_{32}+i W_{23}
\end{array}\right]+\alpha\left(1-\lambda^{2}\right) \mathbf{v}_{3}, \\
& \mathbf{v}_{2}=\overline{\mathbf{v}}_{1}, \quad \mathbf{v}_{3}=\left[\begin{array}{c}
\alpha_{8} \\
-\alpha_{6} \\
\alpha_{4}
\end{array}\right]=-|\boldsymbol{\Lambda}| D\left[\begin{array}{c}
W_{23} \\
-W_{13} \\
W_{12}
\end{array}\right] .
\end{aligned}
$$

Applying $\mathbf{E}^{-1}$ to both sides of (4.5), we obtain

$$
\frac{1}{i \pi} \int_{-a}^{b} \frac{\mathbf{M}(\xi)}{x_{1}-\xi} d \xi+\left[\begin{array}{ccc}
\lambda & 0 & 0 \\
0 & -\lambda & 0 \\
0 & 0 & 0
\end{array}\right] \mathbf{M}\left(x_{1}\right)=i \mathbf{E}^{-1} \boldsymbol{\Lambda}_{1}^{-1} \boldsymbol{\tau}\left(x_{1}\right),
$$

where

$$
\mathbf{M}\left(x_{1}\right)=\mathbf{E}^{-1} \mathbf{B}\left(x_{1}\right) \text {. }
$$

Assuming $\boldsymbol{\tau}\left(x_{1}\right)$ is Hölder continuous, $\mathbf{M}\left(x_{1}\right)$ is obtained from (4.12a). Combining the solution for $\mathbf{M}\left(x_{1}\right)$ with (4.12b), we obtain the solution for $\mathbf{B}\left(x_{1}\right)$,

$$
\begin{aligned}
& \mathbf{B}\left(x_{1}\right)= \frac{i \lambda}{\lambda^{2}-1} \mathbf{E I}_{0} \mathbf{E}^{-1} \boldsymbol{\Lambda}_{1}^{-1} \boldsymbol{\tau}\left(x_{1}\right) \\
&+\frac{1}{\left(\lambda^{2}-1\right) \pi \sqrt{\left(a+x_{1}\right)\left(b-x_{1}\right)}} \\
& \times \int_{-a}^{b} \frac{\sqrt{(a+\xi)(b-\xi)}}{\xi-x_{1}} \mathbf{E}\left(x_{1}, \xi\right) \mathbf{E}^{-1} \Lambda_{1}^{-1} \boldsymbol{\tau}(\xi) d \xi \\
& \text { for }-a<x_{1}<b,
\end{aligned}
$$

with

$$
\begin{aligned}
& \mathbf{I}_{0}=\left[\begin{array}{ccc}
1 & 0 & 0 \\
0 & -1 & 0 \\
0 & 0 & 0
\end{array}\right], \\
& \mathbf{O}\left(x_{1}, \xi\right)=\left[\begin{array}{ccc}
d_{0} & 0 & 0 \\
0 & \bar{d}_{0} & 0 \\
0 & 0 & \left(1-\lambda^{2}\right)
\end{array}\right],
\end{aligned}
$$

where

$$
d_{0}=\left(\frac{\left|a+x_{1}\right|}{\left|b-x_{1}\right|}\right)^{i \gamma_{0}}\left(\frac{|b-\xi|}{|a+\xi|}\right)^{i \gamma_{0}}
$$

and

$$
\gamma_{0}=\frac{1}{2 \pi} \ln \left(\frac{1+\lambda}{1-\lambda}\right) .
$$


Similarly, the interfacial traction vector is given as

$$
\begin{aligned}
\mathbf{t}\left(x_{1}\right)= & \frac{-\operatorname{sgn}\left(x_{1}\right)}{\pi \sqrt{1-\lambda^{2}} \sqrt{\left(x_{1}-b\right)\left(x_{1}+a\right)}} \\
& \times \int_{-a}^{b} \frac{\sqrt{(\xi+a)(b-\xi)}}{\xi-x_{1}} \Lambda_{1} \mathbf{E O}_{1}\left(x_{1}, \xi\right) \mathbf{E}^{-1} \Lambda_{1}^{-1} \boldsymbol{\tau}(\xi) d \xi+\boldsymbol{\tau}\left(x_{1}\right) \\
& \text { for } x_{1}<-a \text { or } x_{1}>b,
\end{aligned}
$$

with

$$
\mathbf{O}_{1}\left(x_{1}, \xi\right)=\left[\begin{array}{ccc}
d_{0} & 0 & 0 \\
0 & \bar{d}_{0} & 0 \\
0 & 0 & \sqrt{1-\lambda^{2}}
\end{array}\right] .
$$

From (4.13) and (4.14), it is seen that $\mathbf{B}\left(x_{1}\right)$ and $\mathbf{t}\left(x_{1}\right)$ have oscillatory singularities, as $x_{1} \rightarrow-a, b$. The necessary and sufficient condition for the vanishing of these oscillatory singularities for any combination of the farfield tractions in the open crack model, is $\lambda=0$, i.e., $\left|\boldsymbol{\Lambda}_{1}\right|=|\boldsymbol{\Lambda}|$, which is equivalent to $\alpha_{4}=\alpha_{6}=\alpha_{8}=0$ or $\Lambda_{2}=0$. The fact that the condition is necessary can be seen by observing the special case of the uniform in-plane farfield tractions, where the existence of nonoscillatory solutions requires $\lambda=0$, except for the trivial case: all farfield tractions are zero. From (4.3d), $\boldsymbol{\Lambda}_{2}=0$ is equivalent to $\mathbf{W}=0$, which is the condition for the existence of nonoscillatory solutions given by Ting [7], and Qu and Bassani [5].

From (4.14a), it is concluded that $\mathbf{E}^{-1} \boldsymbol{\Lambda}_{1}^{-1} \mathbf{t}\left(x_{1}\right)$, a linear combination of the traction vector $\mathbf{t}\left(x_{1}\right)$, always has a nonoscillatory third component at the crack tip. That linear combination corresponds to a linear transformation by $\mathbf{E}^{\mathrm{T}} \boldsymbol{\Lambda}_{1}$ of the local bases at the crack tip. Then in the transformed local coordinates, the traction along the third coordinate direction has no oscillatory singularity. However, unless $\Lambda_{1}$ is diagonal, the transformed bases $\Lambda_{1} \mathbf{v}_{1}, \Lambda_{2}, \Lambda_{1} \mathbf{v}_{3}$ do not necessarily form an orthogonal system.

In the special case of uniform applied farfield stresses, the solutions simplify to

$$
\mathbf{B}\left(x_{1}\right)=\frac{\mathbf{E}}{\sqrt{\left(a+x_{1}\right)\left(b-x_{1}\right)}}\left[\begin{array}{ccc}
\frac{d\left(x_{1}\right)}{\sqrt{1-\lambda^{2}}} & 0 & 0 \\
0 & \frac{\bar{d}\left(x_{1}\right)}{\sqrt{1-\lambda^{2}}} & 0 \\
0 & 0 & x_{1}+\frac{a-b}{2}
\end{array}\right] \mathbf{E}^{-1} \boldsymbol{\Lambda}_{1}^{-1} \boldsymbol{\tau}
$$

and

$$
\mathbf{t}\left(x_{1}\right)=\frac{\operatorname{sgn}\left(x_{1}\right)}{\sqrt{\left(a+x_{1}\right)\left(x_{1}-b\right)}} \mathbf{\Lambda}_{1} \mathbf{E}\left[\begin{array}{ccc}
d\left(x_{1}\right) & 0 & 0 \\
0 & \bar{d}\left(x_{1}\right) & 0 \\
0 & 0 & x_{1}+\frac{a-b}{2}
\end{array}\right] \mathbf{E}^{-1} \boldsymbol{\Lambda}_{1}^{-1} \boldsymbol{\tau},
$$

with

$$
d\left(x_{1}\right)=\left[x_{1}+\frac{a-b}{2}-(a+b) i \gamma_{0}\right]\left|\frac{a+x_{1}}{b-x_{1}}\right|^{i \gamma_{0}} .
$$


4.1. An example. Consider a special case for which $\boldsymbol{\Lambda}$ satisfies $\alpha_{5}=\alpha_{6}=\alpha_{7}=$ $\alpha_{8}=0$, such as a monoclinic material with the $\left(x_{3}=0\right)$-plane as the symmetry plane. Let the farfield tractions $\tau=(S, T, J)$ be uniform. We then have

$$
\boldsymbol{\Lambda}_{1}=\left[\begin{array}{ccc}
\alpha_{1} & \alpha_{3} & 0 \\
\alpha_{3} & \alpha_{2} & 0 \\
0 & 0 & \alpha_{9}
\end{array}\right], \quad \boldsymbol{\Lambda}_{2}=\left[\begin{array}{ccc}
0 & -\alpha_{4} & 0 \\
\alpha_{4} & 0 & 0 \\
0 & 0 & 0
\end{array}\right]
$$

and

$$
\begin{aligned}
& \lambda=\alpha_{4} / \sqrt{\alpha_{1} \alpha_{2}-\alpha_{3}^{2}}, \\
& \mathbf{E}=\left[\begin{array}{ccc}
-\alpha_{3}+i \alpha_{0} & -\alpha_{3}-i \alpha_{0} & 0 \\
\alpha_{1} & \alpha_{1} & 0 \\
0 & 0 & \alpha_{4}
\end{array}\right],
\end{aligned}
$$

with $\alpha_{0}=\sqrt{\alpha_{1} \alpha_{2}-\alpha_{3}^{2}}$.

The dislocation density vector and the interfacial traction vector now become

$$
\begin{aligned}
& B_{1}\left(x_{1}\right)= \frac{T / \sqrt{\alpha_{1} \alpha_{2}-\alpha_{3}^{2}-\alpha_{4}^{2}}}{\sqrt{\left(a+x_{1}\right)\left(b-x_{1}\right)}} \\
& \times\left[-\left(x_{1}+\frac{a-b}{2}\right)\left(\frac{\alpha_{3} \cos t}{\alpha_{0}}+\sin t\right)-\gamma_{0}(a+b)\left(\frac{\alpha_{3} \sin t}{\alpha_{0}}-\cos t\right)\right] \\
&+\frac{S / \sqrt{\alpha_{1} \alpha_{2}-\alpha_{3}^{2}-\alpha_{4}^{2}}}{\sqrt{\left(a+x_{1}\left(b-x_{1}\right)\right.}}\left[\left(x_{1}+\frac{a-b}{2}\right) \frac{\alpha_{2} \cos t}{\alpha_{0}}+\gamma_{0}(a+b) \frac{\alpha_{2} \sin t}{\alpha_{0}}\right], \\
& B_{2}\left(x_{1}\right)= \frac{T / \sqrt{\alpha_{1} \alpha_{2}-\alpha_{3}^{2}-\alpha_{4}^{2}}}{\sqrt{\left(a+x_{1}\right)\left(b-x_{1}\right)}}\left[\left(x_{1}+\frac{a-b}{2}\right) \frac{\alpha_{1} \cos t}{\alpha_{0}}+\gamma_{0}(a+b) \frac{\alpha_{1} \sin t}{\alpha_{0}}\right] \\
&-\frac{S / \sqrt{\alpha_{1} \alpha_{2}-\alpha_{3}^{2}-\alpha_{4}^{2}}}{\sqrt{\left(a+x_{1}\right)\left(b-x_{1}\right)}} \\
& \times\left[\left(x_{1}+\frac{a-b}{2}\right)\left(\frac{\alpha_{3} \cos t}{\alpha_{0}}-\sin t\right)+\gamma_{0}(a+b)\left(\cos t+\frac{\alpha_{3} \sin t}{\alpha_{0}}\right)\right], \\
& B_{3}\left(x_{1}\right)=\frac{J}{\alpha_{9}}\left(x_{1}+\frac{a-b}{2}\right),
\end{aligned}
$$

for $-a<x_{1}<b ;$ and

$$
\begin{array}{r}
t_{1}\left(x_{1}\right)=\frac{\operatorname{sgn}\left(x_{1}\right)}{\sqrt{\left(a+x_{1}\right)\left(x_{1}-b\right)}}\left\{S\left[\left(x_{1}+\frac{a-b}{2}\right) \cos t+\gamma_{0}(a+b) \sin t\right]\right. \\
\left.+\frac{\left(S \alpha_{3}-T \alpha_{1}\right)}{\alpha_{0}}\left[\left(x_{1}+\frac{a-b}{2}\right) \sin t-\gamma_{0}(a+b) \cos t\right]\right\}, \\
t_{2}\left(x_{1}\right)=\frac{\operatorname{sgn}\left(x_{1}\right)}{\sqrt{\left(a+x_{1}\right)\left(x_{1}-b\right)}}\left\{T\left[\left(x_{1}+\frac{a-b}{2}\right) \cos t+\gamma_{0}(a+b) \sin t\right]\right. \\
\left.+\frac{\left(S \alpha_{2}-T \alpha_{3}\right)}{\alpha_{0}}\left[\left(x_{1}+\frac{a-b}{2}\right) \sin t-\gamma_{0}(a+b) \cos t\right]\right\},
\end{array}
$$




$$
t_{3}\left(x_{1}\right)=\frac{\left|x_{1}+\frac{a-b}{2}\right| J}{\sqrt{\left(a+x_{1}\right)\left(x_{1}-b\right)}},
$$

for $x_{1}<-a$ or $x_{1}>b$, where

$$
t=\gamma_{0} \ln \frac{\left|a+x_{1}\right|}{\left|b-x_{1}\right|}, \quad \gamma_{0}=\frac{1}{2 \pi} \ln \frac{\left|\alpha_{4}+\alpha_{0}\right|}{\left|\alpha_{4}-\alpha_{0}\right|} .
$$

Equations $(4.17 \mathrm{a}, \mathrm{b})$ and $(4.18 \mathrm{a}, \mathrm{b})$ are the same as Eqs. (6.5a) and (6.8) in [4]. Furthermore, when $S=T=0$, and $J \neq 0,(4.18 \mathrm{c})$ gives the solution of the interface tractions for pure anti-plane deformation.

4.2. Comments on dimensionless parameters $\alpha_{4}, \alpha_{6}$, and $\alpha_{8}$. We now comment on the dimensionless parameters $\alpha_{4}, \alpha_{6}$, and $\alpha_{8}$. In what follows, we let the halfspaces be monoclinic. From Sec. 5 of [4], for two dissimilar monoclinic materials, both with the $\left(x_{3}=0\right)$-plane as their common plane of symmetry (in which case, $s_{i 4}$ and $s_{i 5}, i=1,2,6$, vanish for both materials), we have

$$
-i \mathbf{A} \mathbf{L}^{-1}=\left[\begin{array}{ccc}
r_{11} & i r_{12} & 0 \\
-i \bar{r}_{12} & r_{22} & 0 \\
0 & 0 & r_{33}
\end{array}\right]
$$

and

$$
\Lambda=\left[\begin{array}{ccc}
b_{1} / e & i d / e & 0 \\
-i \bar{d} / e & b_{2} / e & 0 \\
0 & 0 & f
\end{array}\right]
$$

where

$$
\begin{aligned}
& b_{1}=r_{22+}+r_{22-}, \quad b_{2}=r_{11+}+r_{11-}, \\
& d=r_{12+}-r_{12-}, \quad e=b_{1} b_{2}-d^{2}, \\
& f=\left[r_{33+}+r_{33-}\right]^{-1} .
\end{aligned}
$$

As discussed in Sec. 5 of [4], under rotations $\boldsymbol{\Omega}_{ \pm}$from the $x_{i}$-coordinates into the (rectangular Cartesian) $x_{i}^{*}$-coordinates, we have

$$
\mathbf{\Lambda}=-i\left[\mathbf{\Omega}_{+}^{T} \overline{\mathbf{A}}_{+}^{*} \overline{\mathbf{L}}_{+}^{*-1} \mathbf{\Omega}_{+}-\mathbf{\Omega}_{-}^{T} \mathbf{A}_{-}^{*} \mathbf{L}_{-}^{*-1} \mathbf{\Omega}_{-}\right]^{-1},
$$

where

$$
\boldsymbol{\Omega}_{ \pm}=\left[\begin{array}{ccc}
\cos \theta_{ \pm} & \sin \theta_{ \pm} & 0 \\
-\sin \theta_{ \pm} & \cos \theta_{ \pm} & 0 \\
0 & 0 & 1
\end{array}\right] \text {. }
$$

Provided that $-i \mathbf{A}_{ \pm}^{*} \mathbf{L}_{ \pm}^{*-1}$ have the form of (4.19a), we obtain the following expression for $\boldsymbol{\Lambda}$

where

$$
\Lambda=\left[\begin{array}{ccc}
b_{1} / e & i d / e & 0 \\
-i \bar{d} / e & b_{2} / e & 0 \\
0 & 0 & f
\end{array}\right]
$$

$$
\begin{aligned}
b_{1}= & {\left[r_{11}^{*} \sin ^{2} \theta+r_{22}^{*} \cos ^{2} \theta+\operatorname{Im}\left(r_{12}^{*}\right) \sin 2 \theta\right]_{+} } \\
& +\left[r_{11}^{*} \sin ^{2} \theta+r_{22}^{*} \cos ^{2} \theta+\operatorname{Im}\left(r_{12}^{*}\right) \sin 2 \theta\right]_{-},
\end{aligned}
$$




$$
\begin{gathered}
b_{2}=\left[r_{11}^{*} \cos ^{2} \theta+r_{22}^{*} \sin ^{2} \theta-\operatorname{Im}\left(r_{12}^{*}\right) \sin 2 \theta\right]_{+} \\
+\left[r_{11}^{*} \cos ^{2} \theta+r_{22}^{*} \sin ^{2} \theta-\operatorname{Im}\left(r_{12}^{*}\right) \sin 2 \theta\right]_{-}, \\
d=\left[\operatorname{Re}\left(r_{12}^{*}\right)_{+}-\operatorname{Re}\left(r_{12}^{*}\right)_{-}\right]+\frac{i}{2}\left\{\left[\left(r_{11}^{*}-r_{22}^{*}\right) \sin 2 \theta-2 \operatorname{Im}\left(r_{12}^{*}\right) \cos 2 \theta\right]_{+}\right. \\
\left.+\left[\left(r_{11}^{*}-r_{22}^{*}\right) \sin 2 \theta-2 \operatorname{Im}\left(r_{12}^{*}\right) \cos 2 \theta\right]_{-}\right\}, \\
e=b_{1} b_{2}-|d|^{2},
\end{gathered}
$$

and

$$
f=\left(r_{33+}^{*}+r_{33-}^{*}\right)^{-1} .
$$

Therefore, it can be seen that

$$
\begin{aligned}
& \alpha_{4}=\operatorname{Re}\left(r_{12}^{*}\right)_{+}-\operatorname{Re}\left(r_{12}^{*}\right)_{-}, \\
& \alpha_{6}=\alpha_{8}=0,
\end{aligned}
$$

which are invariant under the rotations $\boldsymbol{\Omega}_{ \pm}$. In other words, if two monoclinic materials are matched such that $\operatorname{Re}\left(r_{12+}\right)=\operatorname{Re}\left(r_{12-}\right)$, then $\alpha_{4}=\alpha_{6}=\alpha_{8}=0$, provided that they have the common symmetry plane $x_{3}=0$.

The evaluation of $r_{i j}$ for monoclinic materials is given by Suo [6] and Ting [9], which may also be obtained by the method described in Sec. 5 of [4]. Specifically, we have

$$
\operatorname{Re}\left(r_{12}^{*}\right)=S_{12}^{*}-S_{11}^{*} \operatorname{Re}\left(p_{1} p_{2}\right),
$$

where $p_{1}$ and $p_{2}$ are the roots of the equation

$$
p^{4} S_{11}^{*}-2 p^{3} S_{16}^{*}+p^{2}\left(2 S_{12}^{*}+S_{66}^{*}\right)-2 p S_{26}^{*}+S_{22}^{*}=0,
$$

with $\operatorname{Im} p_{i}>0, i=1,2$, and $S_{m n}, m, n=1,2,4,5,6$, defined in terms of the elastic compliance matrix $\left[s_{k j}\right], k, j=1,2, \ldots, 6$, by $s_{m n}=s_{m n}-s_{m 3} s_{n 3} / s_{33}$. For plane problems, the matrix $\left[S_{m n}\right]$ is the inverse of the elastic stiffness matrix $\left[c_{m n}\right], m, n=1,2,4,5,6$.

As a special case, let both materials be orthotropic. Then we can evaluate $r_{i j}$ as follows, providing that the symmetry directions of both materials coincide with the coordinate directions,

$$
\begin{aligned}
& r_{11}=\frac{1}{\left(c_{12}+c_{0}\right)}\left[\frac{c_{22}\left(2 c_{0}-c\right)}{c_{66}\left(c_{0}-c_{12}\right)}\right]^{1 / 2}, \\
& r_{22}=\left(\frac{c_{11}}{c_{22}}\right)^{1 / 2} r_{11}, \quad r_{12}=\left(c_{12}+c_{0}\right)^{-1},
\end{aligned}
$$

with

$$
c_{0}=\left(c_{11} c_{22}\right)^{1 / 2}, \quad c=c_{0}-c_{12}-2 c_{66},
$$

as given in Sec. 5 of [4]. 
So, if two monoclinic materials are matched such that

$$
\left[s_{12}-s_{11} \operatorname{Re}\left(p_{1} p_{2}\right)\right]_{+}=\left[s_{12}-s_{11} \operatorname{Re}\left(p_{1} p_{2}\right)\right]_{-},
$$

or two orthotropic materials are matched such that

$$
\left(c_{0}+c_{12}\right)_{+}=\left(c_{0}+c_{12}\right)_{-},
$$

then $\alpha_{4}=\alpha_{6}=\alpha_{8}=0$, provided that they have the common symmetry plane $x_{3}=0$, or the symmetry directions of both materials coincide with the coordinates, respectively. The validity of Eqs. $(4.25 \mathrm{a}, \mathrm{b})$ is invariant under the rotation of each material about the $x_{3}$-axis. The above conclusions are direct consequences of the discussion in Sec. 5 of [4] and are consistent with the results in Ting [8, 9].

5. The Comninou model. We follow Gautesen and Dundurs [3] to symmetrize the interval of the integration in (3.3) as follows:

$$
\begin{aligned}
& x_{1}=L \frac{s-\gamma}{1-\gamma s}, \quad \xi=L \frac{\eta-\gamma}{1-\gamma \eta}, \\
& B_{j}\left(x_{1}\right)=(1-\gamma s)^{2} \Phi_{j}(s), \quad j=1,2,3,
\end{aligned}
$$

where

$$
\gamma=\frac{L(a-b)}{L^{2}-a b+\sqrt{\left(L^{2}-a^{2}\right)\left(L^{2}-b^{2}\right)}} .
$$

Equation (3.3) is now transformed to

$$
\left[\begin{array}{ccc}
1 & 0 & 0 \\
0 & H\left(c^{2}-s^{2}\right) & 0 \\
0 & 0 & 1
\end{array}\right]\left\{\frac{1}{\pi} \operatorname{Re}\left[\frac{\boldsymbol{\Lambda}}{s-0 i}\right] * \boldsymbol{\Phi}(s)+\frac{\boldsymbol{\tau}}{(1-\gamma s)^{2}}\right\}=0, \quad \text { for }|s|<1 .
$$

Conditions (3.4a-f) then become

$$
\begin{aligned}
& \Phi_{1}(s)=\Phi_{3}(s)=0, \quad \text { for }|s|>1, \quad \Phi_{2}(s)=0, \quad \text { for }|s|>c, \\
& \int_{-1}^{1} \Phi_{1}(s) d s=\int_{-1}^{1} \Phi_{3}(s) d s=0, \quad \int_{-c}^{c} \Phi_{2}(s) d s=0,
\end{aligned}
$$

where

$$
c=\frac{a \sqrt{L^{2}-b^{2}}+b \sqrt{L^{2}-a^{2}}}{L\left[\sqrt{L^{2}-b^{2}}+\sqrt{L^{2}-a^{2}}\right]} .
$$

Equation (5.2) can be reduced to

$\left[\begin{array}{ccc}1 & 0 & 0 \\ 0 & H\left(c^{2}-s^{2}\right) & 0 \\ 0 & 0 & 1\end{array}\right]\left\{\frac{-\boldsymbol{\Lambda}_{1}}{\pi} \int_{-1}^{1} \frac{\boldsymbol{\Phi}(\xi)}{\xi-s} d \xi+\boldsymbol{\Lambda}_{2} \boldsymbol{\Phi}(s)+\frac{\boldsymbol{\tau}}{(1-\gamma s)^{2}}\right\}=0$,

$$
\text { for }|s|<1 \text {, }
$$


which, in component form becomes

$$
\begin{aligned}
& \alpha_{4} \Phi_{2}(s) H\left(c^{2}-s^{2}\right)+\alpha_{6} \Phi_{3}(s)+\frac{\alpha_{1}}{\pi} \int_{-1}^{1} \frac{\Phi_{1}(\xi)}{\xi-s} d \xi \\
& +\frac{\alpha_{3}}{\pi} \int_{-c}^{c} \frac{\Phi_{2}(\xi)}{\xi-s} d \xi+\frac{\alpha_{5}}{\pi} \int_{-1}^{1} \frac{\Phi_{3}(\xi)}{\xi-s} d \xi=\frac{S}{(1-\gamma s)^{2}}, \quad \text { for }|s|<1, \\
& -\alpha_{4} \Phi_{1}(s)+\alpha_{8} \Phi_{3}(s)+\frac{\alpha_{3}}{\pi} \int_{-1}^{1} \frac{\Phi_{1}(\xi)}{\xi-s} d \xi \\
& +\frac{\alpha_{2}}{\pi} \int_{-c}^{c} \frac{\Phi_{2}(\xi)}{\xi-s} d \xi+\frac{\alpha_{7}}{\pi} \int_{-1}^{1} \frac{\Phi_{3}(\xi)}{\xi-s} d \xi=\frac{T}{(1-\gamma s)^{2}}, \quad \text { for }|s|<c, \\
& -\alpha_{6} \Phi_{1}(s)-\alpha_{8} \Phi_{2}(s) H\left(c^{2}-s^{2}\right)+\frac{\alpha_{5}}{\pi} \int_{-1}^{1} \frac{\Phi_{1}(\xi)}{\xi-s} d \xi \\
& +\frac{\alpha_{7}}{\pi} \int_{-c}^{c} \frac{\Phi_{2}(\xi)}{\xi-s} d \xi+\frac{\alpha_{9}}{\pi} \int_{-1}^{1} \frac{\Phi_{3}(\xi)}{\xi-s} d \xi=\frac{J}{(1-\gamma s)^{2}}, \quad \text { for }|s|<1
\end{aligned}
$$

As discussed in [4], we seek the solution $\Phi_{2}(s)$ in the class of functions, which is Hölder continuous on $[-c, c]$. Then the Poincaré-Bertrand commutation formula applies. From $(5.5 \mathrm{a}, \mathrm{c})$, we solve for $\Phi_{1}(s)$ and $\Phi_{3}(s)$ in terms of $\Phi_{2}(s)$ and $\int_{-c}^{c} \Phi_{2}(\xi) /(\xi-s) d \xi$. Then, substituting these solutions into Eq. (5.5b), we obtain a single equation for $\Phi_{2}(s)$. After lengthy calculation, we arrive at

$$
\begin{aligned}
\Phi_{1}(s)= & {\left[\frac{\alpha_{5} \alpha_{7}+\alpha_{6} \alpha_{8}-\alpha_{3} \alpha_{9}}{\alpha_{1} \alpha_{9}-\alpha_{5}^{2}-\alpha_{6}^{2}}\right] \Phi_{2}(s) } \\
& +\left[\frac{\alpha_{4} \alpha_{9}+\alpha_{5} \alpha_{8}-\alpha_{6} \alpha_{7}}{\pi\left(\alpha_{1} \alpha_{9}-\alpha_{5}^{2}-\alpha_{6}^{2}\right)}\right] \operatorname{Re}\left[\int_{-c}^{c} \frac{\Phi_{2}(\xi)}{\xi-s}\left(\frac{1+\xi}{1+s}\right)^{1-m}\left(\frac{1-\xi}{1-s}\right)^{m} d \xi\right] \\
& +\left[\frac{\left.\alpha_{5} \alpha_{6} \alpha_{7}+\alpha_{1} \alpha_{8} \alpha_{9}-\alpha_{8} \alpha_{5}^{2}-\alpha_{3} \alpha_{6} \alpha_{9}\right]}{\pi \alpha_{00}\left(\alpha_{1} \alpha_{9}-\alpha_{5}^{2}-\alpha_{6}^{2}\right)}\right] \\
& +\operatorname{Im}\left[\int_{-c}^{c} \frac{\Phi_{2}(\xi)}{\xi-s}\left(\frac{1+\xi}{1+s}\right)^{1-m}\left(\frac{1-\xi}{1-s}\right)^{m} d \xi\right] \\
& \left.+\frac{\alpha_{9} S-\alpha_{5} J}{\alpha_{00} \sqrt{\alpha}_{1} \alpha_{9}-\alpha_{5}^{2}-\alpha_{6}^{2}}\right] \operatorname{Re}\left\{\frac{d}{d \gamma}\left[\left(\frac{1+\gamma}{1+s}\right)^{1-m}\left(\frac{1-\gamma}{1-s}\right)^{m} \frac{1}{1-s \gamma}\right]\right\} \\
& \sqrt{\alpha_{1} \alpha_{9}-\alpha_{5}^{2}-\alpha_{6}^{2}} \operatorname{Im}\left\{\frac{d}{d \gamma}\left[\left(\frac{1+\gamma}{1+s}\right)^{1-m}\left(\frac{1-\gamma}{1-s}\right)^{m} \frac{1}{1-s \gamma}\right]\right\}, \quad(5.6 a)
\end{aligned}
$$




$$
\begin{aligned}
\Phi_{3}(s)= & {\left[\frac{\alpha_{4} \alpha_{6}+\alpha_{3} \alpha_{5}-\alpha_{1} \alpha_{7}}{\alpha_{1} \alpha_{9}-\alpha_{5}^{2}-\alpha_{6}^{2}}\right] \Phi_{2}(s) } \\
& +\left[\frac{\alpha_{3} \alpha_{6}-\alpha_{4} \alpha_{5}-\alpha_{1} \alpha_{8}}{\pi\left(\alpha_{1} \alpha_{9}-\alpha_{5}^{2}-\alpha_{6}^{2}\right)}\right] \operatorname{Re}\left[\int_{-c}^{c} \frac{\Phi_{2}(\xi)}{\xi-s}\left(\frac{1+\xi}{1+s}\right)^{1-m}\left(\frac{1-\xi}{1-s}\right)^{m} d \xi\right] \\
& \left.+\left[\frac{\alpha_{1} \alpha_{4} \alpha_{9}-\alpha_{4} \alpha_{5}^{2}-\alpha_{1} \alpha_{6} \alpha_{7}+\alpha_{3} \alpha_{5} \alpha_{6}}{\pi \alpha_{00}\left(\alpha_{1} \alpha_{9}-\alpha_{5}^{2}-\alpha_{6}^{2}\right)}\right]^{1-m}\left(\frac{1-\xi}{1-s}\right)^{m} d \xi\right] \\
& +\operatorname{Im}\left[\int_{-c}^{c} \frac{\Phi_{2}(\xi)}{\xi-s}\left(\frac{1+\xi}{1+s}\right)^{1-\alpha_{1} J-\alpha_{5} S}\right] \operatorname{Re}\left\{\frac{d}{d \gamma}\left[\left(\frac{1+\gamma}{1+s}\right)^{1-m}\left(\frac{1-\gamma}{1-s}\right)^{m} \frac{1}{1-s \gamma}\right]\right\} \\
& \left.+\frac{\left.\alpha_{00} \sqrt{\alpha_{1} \alpha_{9}-\alpha_{5}^{2}-\alpha_{6}^{2}}\right]}{\sqrt{\alpha_{1} \alpha_{9}-\alpha_{5}^{2}-\alpha_{6}^{2}} \operatorname{Im}\left\{\frac{d}{d \gamma}\left[\left(\frac{1+\gamma}{1+s}\right)^{1-m}\left(\frac{1-\gamma}{1-s}\right)^{m} \frac{1}{1-s \gamma}\right]\right\}, \quad(5.6}\right)
\end{aligned}
$$

where $\alpha_{00}=\sqrt{\alpha_{1} \alpha_{9}-\alpha_{5}^{2}}$, and

$$
m=\frac{1}{2}+\frac{i}{2 \pi} \ln \left|\frac{\alpha_{00}+\alpha_{6}}{\alpha_{00}-\alpha_{6}}\right| .
$$

The single equation for $\Phi_{2}(s)$ then is

$$
\begin{aligned}
& \int_{-c}^{c}\left\{1-a_{2} \operatorname{Re}\left[\left(\frac{1+\xi}{1+s}\right)^{1-m}\left(\frac{1-\xi}{1-s}\right)^{m}\right]\right\} \frac{\Phi_{2}(\xi)}{\xi-s} d \xi \\
& \quad=a_{3} \frac{d}{d \gamma}\left[\frac{\gamma}{1-\gamma s}\right]+a_{4} \operatorname{Re}\left\{\frac{d}{d \gamma}\left[\left(\frac{1+\gamma}{1+s}\right)^{1-m}\left(\frac{1-\gamma}{1-s}\right)^{m} \frac{1}{1-s \gamma}\right]\right\} \\
& \quad+a_{5} \operatorname{Im}\left\{\frac{d}{d \gamma}\left[\left(\frac{1+\gamma}{1+s}\right)^{1-m}\left(\frac{1-\gamma}{1-s}\right)^{m} \frac{1}{1-s \gamma}\right]\right\}, \quad \text { for }|s|<c,
\end{aligned}
$$

with

$$
\begin{aligned}
& a_{2}= \frac{1}{\left|\Lambda_{1}\right|}\left[\alpha_{00}^{2}\left(\alpha_{4} \alpha_{5}+\alpha_{1} \alpha_{8}-\alpha_{3} \alpha_{6}\right)^{2}+\left(\alpha_{00}^{2} \alpha_{4}-\alpha_{1} \alpha_{6} \alpha_{7}+\alpha_{3} \alpha_{5} \alpha_{6}\right)^{2}\right], \\
& a_{3}= \frac{\pi \alpha_{1}}{\left|\Lambda_{1}\right|}\left[\left(\alpha_{1} \alpha_{9}-\alpha_{5}^{2}\right) T-\left(\alpha_{3} \alpha_{9}-\alpha_{5} \alpha_{7}\right) S-\left(\alpha_{1} \alpha_{7}-\alpha_{3} \alpha_{5}\right) J\right], \\
& a_{4}= \frac{-\pi \alpha_{1} \alpha_{00}}{\left|\Lambda_{1}\right|}\left[\left(\alpha_{1} \alpha_{8}+\alpha_{4} \alpha_{5}-\alpha_{3} \alpha_{6}\right) J-\left(\alpha_{4} \alpha_{9}+\alpha_{5} \alpha_{8}-\alpha_{6} \alpha_{7}\right) S\right], \\
& a_{5}= \frac{-\pi \alpha_{1}}{\left|\Lambda_{1}\right|}\left\{\left[\alpha_{1}\left(\alpha_{4} \alpha_{9}-\alpha_{6} \alpha_{7}\right)+\alpha_{5}\left(\alpha_{3} \alpha_{6}-\alpha_{4} \alpha_{5}\right)\right] J\right. \\
&\left.\quad-\left[\alpha_{9}\left(\alpha_{3} \alpha_{6}-\alpha_{1} \alpha_{8}\right)+\alpha_{5}\left(\alpha_{5} \alpha_{8}-\alpha_{6} \alpha_{7}\right)\right] S\right\} .
\end{aligned}
$$

From (5.6c), it is clear that if $\alpha_{6}=0$, then $m=\frac{1}{2}$. Therefore, the solutions $\Phi_{j}(s), j=1,2,3$, have no oscillatory singularities as $s \rightarrow \pm 1$. Conversely, we prove that if $\alpha_{6} \neq 0$, then for certain combinations of the farfield tractions $S$, 
$T$, and $J$, the functions $\Phi_{1}(s)$ or $\Phi_{3}(s)$, or both have oscillatory singularities. Therefore, $\alpha_{6}=0$ is the necessary and sufficient condition under which oscillatory singularities do not exist for any combinations of the farfield tractions.

Assume $S=J=0, T \neq 0$. Then as $s \rightarrow \pm 1$, we can express $\Phi_{1}(s)$ and $\Phi_{3}(s)$ as

$$
\begin{aligned}
\Phi_{1}(s) \approx & d_{11} \operatorname{Re}\left[\int_{-c}^{c} \frac{\Phi_{2}(\xi)}{\xi-s}\left(\frac{1+\xi}{1+s}\right)^{1-m}\left(\frac{1-\xi}{1-s}\right)^{m} d \xi\right] \\
& +d_{12} \operatorname{Im}\left[\int_{-c}^{c} \frac{\Phi_{2}(\xi)}{\xi-s}\left(\frac{1+\xi}{1+s}\right)^{1-m}\left(\frac{1-\xi}{1-s}\right)^{m} d \xi\right], \\
\Phi_{3}(s) \approx & d_{31} \operatorname{Re}\left[\int_{-c}^{c} \frac{\Phi_{2}(\xi)}{\xi-s}\left(\frac{1+\xi}{1+s}\right)^{1-m}\left(\frac{1-\xi}{1-s}\right)^{m} d \xi\right] \\
& +d_{32} \operatorname{Im}\left[\int_{-c}^{c} \frac{\Phi_{2}(\xi)}{\xi-s}\left(\frac{1+\xi}{1+s}\right)^{1-m}\left(\frac{1-\xi}{1-s}\right)^{m} d \xi\right],
\end{aligned}
$$

where $d_{11}, d_{12}, d_{31}, d_{32}$ denote the corresponding coefficients in (5.6a, b). We write

$$
\int_{-c}^{c} \frac{\Phi_{2}(\xi)}{\xi-s}\left(\frac{1+\xi}{1+s}\right)^{1-m}\left(\frac{1-\xi}{1-s}\right)^{m} d \xi=h_{1}(s)+i h_{2}(s)
$$

where $h_{1}(s)$ and $h_{2}(s)$ are real functions with singularities as $s \rightarrow \pm 1$. Then

$$
\begin{aligned}
\Phi_{1}(s) \approx & d_{11} \operatorname{Re}\left[(1+s)^{m-1}(1-s)^{-m}\left(h_{1}(s)+i h_{2}(s)\right)\right] \\
& +d_{12} \operatorname{Im}\left[(1+s)^{m-1}(1-s)^{-m}\left(h_{1}(s)+i h_{2}(s)\right)\right] .
\end{aligned}
$$

As $s \rightarrow \pm 1$, nonoscillatory solutions of $\Phi_{1}(s)$ require

$$
d_{11} h_{1}+d_{12} h_{2}=0, \quad d_{12} h_{1}-d_{11} h_{2}=0 .
$$

Since $\Phi_{2}(s)$ is not identically zero, $h_{1}(s)$ and $h_{2}(s)$ are not identically zero. It follows that constants $d_{11}, d_{12}$ must be zero. The same comments apply to $\Phi_{3}(s)$. Therefore, if $d_{11}, d_{12}, d_{31}, d_{32}$ are not all zero, then, from (5.6a, b), $S=J=0$, and $T \neq 0$, either $\Phi_{1}(s)$ or $\Phi_{3}(s)$ will have oscillatory singularities, as $s \rightarrow \pm 1$. Otherwise, if $d_{11}, d_{12}, d_{31}, d_{32}$ are all zero, it is easy to see from $(5.6 \mathrm{a}, \mathrm{b})$ with $J=0$ or $S=0$, that either $\Phi_{1}(s)$ or $\Phi_{3}(s)$ will have oscillatory singularities as $s \rightarrow \pm 1$.

When $\alpha_{6}=0$, Eq. (5.7a) becomes

$$
\begin{aligned}
\int_{-c}^{c} & {\left[1-a_{2}\left(\frac{1-\xi^{2}}{1-s^{2}}\right)^{1 / 2}\right] \frac{\Phi_{2}(\xi)}{\xi-s} d \xi } \\
& =a_{3} \frac{d}{d \gamma}\left[\frac{\gamma}{1-\gamma s}\right]+a_{4} \frac{d}{d \gamma}\left[\left(\frac{1-\gamma^{2}}{1-s^{2}}\right)^{1 / 2} \frac{1}{1-\gamma s}\right], \quad \text { for }|s|<c,
\end{aligned}
$$

where $a_{2}>0$. It is interesting to note that $(5.10)$ is in exactly the same form as (7.9) of [4] or (2.18) in Gautesen and Dundurs [3], which have been thoroughly 
discussed by these authors. Therefore, for $\alpha_{6}=0$, the desired solutions follow readily. Furthermore, from $(4.11 \mathrm{e})$, it is easy to see that $\alpha_{6}$ can be expressed in the elements of matrices $\mathbf{D}$ and $\mathbf{W}$, as

$$
\alpha_{6}=D_{22} W_{13}-D_{21} W_{23}-D_{23} W_{12},
$$

where $\mathbf{D}$ and $\mathbf{W}$ are defined by Ting [7].

However, when $\alpha_{6} \neq 0$, it appears difficult to obtain an analytical solution for (5.7a).

In order to eliminate possible oscillatory singularities when $\alpha_{6} \neq 0$, we may consider a modification of the Comninou model, by assuming that the crack has no sliding in the $x_{2}$ - and $x_{3}$-directions over a small contact zone near its ends. In this modified model, a single equation for $\Phi_{2}(s)$ is obtained, which has a rather complicated form with no transparent solutions; see Appendix. We note, however, that there seems to be little physical justification for requiring no sliding over a small region close to the crack tips, unless the contact is frictional.

Appendix. In this appendix, we give a modified Comninou model, which eliminates possible oscillatory singularities, when $\alpha_{6} \neq 0$.

As in Sec. 3.2, let the interface crack on the $\left(x_{1}, x_{3}\right)$-plane extend from $-L$ to $L$, with the frictionless contact zones $(-L, a)$ and $(b, L)$. Assume that the crack is open and traction-free over the interval $(-a, b)$. Unlike in the Comninou model, which we considered in Sec. 5, where sliding in the $x_{1}$ - and $x_{3}$-directions is allowed over the whole interval $(-L, L)$, we now assume that over the contact zones $(-L,-a)$ and $(b, L)$ there is no sliding in the $x_{3}$-direction. Then, under uniform applied farfield tractions $\tau=(S, T, J)$, the tangential tractions in the $x_{1}$-direction vanish over the interval $(-L, L)$, and the normal tractions in the $x_{2}$-direction and the tangential tractions in the $x_{3}$-direction vanish over the interval $(-a, b)$.

Therefore, after using transformation (5.1), the following equation for $\boldsymbol{\Phi}(s)$ is obtained,

$$
\begin{array}{ccc}
{\left[\begin{array}{ccc}
1 & 0 & 0 \\
0 & H\left(c^{2}-s^{2}\right) & 0 \\
0 & 0 & H\left(c^{2}-s^{2}\right)
\end{array}\right]\left\{\frac{-\boldsymbol{\Lambda}_{1}}{\pi} \int_{-1}^{1} \frac{\boldsymbol{\Phi}(\xi)}{\xi-s} d \xi+\boldsymbol{\Lambda}_{2} \boldsymbol{\Phi}(s)+\frac{\boldsymbol{\tau}}{(1-\gamma s)^{2}}\right\}=0} \\
\text { for }|s|<1,
\end{array}
$$

subject to conditions

$$
\begin{aligned}
& \Phi_{1}(s)=0, \quad \text { for }|s|>1, \\
& \Phi_{2}(s)=\Phi_{3}(s)=0, \text { for }|s|>c,
\end{aligned}
$$

and

$$
\begin{aligned}
& \int_{-c}^{c} \Phi_{1}(s) d s=0, \\
& \int_{-1}^{1} \Phi_{2}(s) d s=\int_{-1}^{1} \Phi_{3}(s) d s=0,
\end{aligned}
$$

where $c$ is defined by $(5.3 \mathrm{~g})$. 
Under certain smoothness assumptions for $\Phi_{i}(s), i=1,2,3$, similar to those discussed in Sec. 5, from (A.1), $\Phi_{1}(s)$ and $\Phi_{2}(s)$ may be obtained as follows:

$$
\Phi_{2}(s)=l_{1} \Phi_{3}(s)+\frac{l_{2}}{\pi i}\left(\frac{c+s}{c-s}\right)^{p} \int_{-c}^{c}\left(\frac{c-\eta}{c+\eta}\right) \frac{\Phi_{3}(\eta)}{\eta-s} d \eta+f_{12}(s),
$$

where

$$
\begin{aligned}
l_{1} & =\left(a d_{1}+b d_{2}\right) /\left(a^{2}+b^{2}\right), \quad l_{2}=\left(a d_{2}-b d_{1}\right) /\left(a^{2}+b^{2}\right), \\
f_{12}(s) & =-\frac{1}{\pi\left(a^{2}+b^{2}\right)} \int_{-c}^{c} \frac{\alpha_{4} f_{1}(\xi)+\alpha_{6} f_{2}(\xi)}{\xi-s} d \xi,
\end{aligned}
$$

with

$$
\begin{aligned}
& a=\alpha_{4}\left(\alpha_{1} \alpha_{8}+\alpha_{4} \alpha_{5}-\alpha_{3} \alpha_{6}\right), \\
& b=\alpha_{6}\left(\alpha_{1} \alpha_{2}-\alpha_{3}^{2}\right)+\alpha_{4}\left(\alpha_{3} \alpha_{5}-\alpha_{1} \alpha_{7}\right), \\
& d_{1}=-a \alpha_{6} / \alpha_{4}, \quad d_{2}=\alpha_{4}\left(\alpha_{1} \alpha_{9}-\alpha_{5}^{2}\right)+\alpha_{6}\left(\alpha_{3} \alpha_{5}-\alpha_{1} \alpha_{7}\right), \\
& f_{1}(s)=\alpha_{5} \frac{S}{(1-\gamma s)^{2}}-\alpha_{1} \frac{J}{(1-\gamma s)^{2}}-\alpha_{6} S_{1} \frac{d}{d \gamma}\left[\frac{\sqrt{1-\gamma^{2}}}{1-\gamma s}\right], \\
& f_{2}(s)=-\alpha_{3} \frac{S}{(1-\gamma s)^{2}}+\alpha_{1} \frac{T}{(1-\gamma s)^{2}}-\alpha_{4} S \frac{d}{d \gamma}\left[\frac{\sqrt{1-\gamma^{2}}}{1-\gamma s}\right],
\end{aligned}
$$

and

$$
p=\theta / \pi, \quad \operatorname{tg} \theta=b / a
$$

(ii) $\Phi_{1}(s)=-\frac{\alpha_{3}}{\alpha_{1}} \Phi_{2}(s)-\frac{\alpha_{5}}{\alpha_{1}} \Phi_{3}(s)+\frac{\alpha_{4}}{\alpha_{1} \pi \sqrt{1-s^{2}}} \int_{-c}^{c} \frac{\left(1-\xi^{2}\right)^{1 / 2} \Phi_{2}(\xi)}{\xi-s} d \xi$

$$
+\frac{\alpha_{6}}{\alpha_{1} \pi \sqrt{1-s^{2}}} \int_{-c}^{c} \frac{\left(1-\xi^{2}\right)^{1 / 2} \Phi_{3}(\xi)}{\xi-s} d \xi+\frac{\tau_{1}}{\alpha_{1}} \frac{d}{d \gamma}\left[\frac{\sqrt{1-\gamma^{2}}}{1-\gamma s}\right]
$$

(iii) $\Phi_{3}(s)$ satisfies the single equation

$$
\begin{aligned}
& q_{1} \Phi_{3}(s)+q_{2} \int_{-c}^{c} \frac{\Phi_{3}(\xi)}{\xi-s} d \xi+\frac{q_{3}}{\sqrt{1-s^{2}}} \int_{-c}^{c} \frac{\sqrt{1-\xi^{2}}}{\xi-s} \Phi_{3}(\xi) d \xi \\
& +q_{4}\left(\frac{c+s}{c-s}\right)^{p} \int_{-c}^{c}\left(\frac{c-\xi}{c+\xi}\right)^{p} \frac{\Phi_{3}(\xi)}{\xi-s} d \xi \\
& \quad+\frac{q_{5}}{\sqrt{1-s^{2}}} \int_{-c}^{c}\left(\frac{c-\eta}{c+\eta}\right)^{p} \Phi_{3}(\eta) \int_{-c}^{c} \frac{\sqrt{1-\xi^{2}}}{(\xi-s)(\eta-\xi)}\left(\frac{c-\xi}{c-\xi}\right)^{p} d \xi d \eta+q_{6}=0
\end{aligned}
$$


where

$$
\begin{aligned}
& q_{1}=\frac{a}{\alpha_{4}}+l_{2}\left(\alpha_{1} \alpha_{2}-\alpha_{3}^{2}-\alpha_{4}^{2}\right), \\
& q_{2}=\frac{1}{\pi}\left[\left(\alpha_{1} \alpha_{2}-\alpha_{3}^{2}\right)\left(l_{1}+\frac{a}{b} l_{2}\right)+\left(\alpha_{1} \alpha_{7}-\alpha_{3} \alpha_{5}\right)\right], \\
& q_{3}=-\left(\alpha_{4}^{2}+\alpha_{4} \alpha_{6}\right), \quad q_{4}=-\frac{a l_{2}\left(\alpha_{1} \alpha_{2}-\alpha_{3}^{2}\right)}{\pi b}, \quad q_{5}=-\frac{\alpha_{4}^{2} l_{2}}{\pi}, \\
& q_{6}=-f_{2}(s)+\frac{\alpha_{1} \alpha_{2}-\alpha_{3}^{2}}{\pi} \int_{-c}^{c} \frac{f_{12}(\xi)}{\xi-s} d \xi-\frac{\alpha_{4}^{2}}{\pi \sqrt{1-s^{2}}} \int_{-c}^{c} \frac{\sqrt{1-\xi^{2}} f_{12}(\xi)}{\xi-s} d \xi .
\end{aligned}
$$

The solution for Eq. (A.5) is not available yet. But, it can be seen that the solutions of $\Phi_{i}(s), i=1,2,3$, do not have an oscillatory singularity as $s \rightarrow \pm 1$.

Acknowledgment. This work has been supported by the U.S. Army Research Office under Contract No. DAAL-03-86-K-0169 to the University of California, San Diego.

\section{REFERENCES}

[1] M. Comninou, The interface crack, ASME J. Appl. Mech. 44, 631-636 (1977)

[2] A. K. Gautesen and J. Dundurs, The interface crack in a tension field, ASME J. Appl. Mech. 54, 93-98 (1987)

[3] A. K. Gautesen and J. Dundurs, The interface crack under combined loading, ASME J. Appl. Mech. 55, 580-586 (1988)

[4] L. Ni and S. Nemat-Nasser, Interface crack in anisotropic dissimilar materials: an analytic solution, J. Mech. Phys. Solids 39, 113-144 (1991)

[5] J. Qu and J. L. Bassani, Crack on bimaterial and bicrystal interfaces, J. Mech. Phys. Solids 37, 417-433 (1989)

[6] Z. Suo, Singularities, interfaces and cracks in dissimilar anisotropic media, Proc. Roy. Soc. London Ser. A 427, 331-358 (1990)

[7] T. C. T. Ting, Explicit solution and invariance of the singularities at an interface crack in anisotropic composites, Internat. J. Solids and Structures 22, 965-983 (1986)

[8] T. C. T. Ting, Interface cracks in anisotropic bimaterials, J. Mech. Phys. Solids 38, 505-513 (1990)

[9] T. C. T. Ting, Barnett-Lothe tensors and their associated tensors for monoclinic materials with the symmetry plane at $x_{3}=0, \mathrm{~J}$. Elasticity (to appear)

[10] J. R. Willis, Fracture mechanics of interface cracks, J. Mech. Phys. Solids 19, 353-368 (1971)

[11] K.-C. Wu, Stress intensity factor and energy release rate for interface cracks between dissimilar anisotropic materials, ASME J. Appl. Mech. 57, 894-900 (1990) 